\title{
天然药物小檗碱的化学合成研究进展
}

\author{
严锡飞 $a$ 郑剑峰*,a李卫东*,a,b \\ ( ${ }^{a}$ 西南交通大学生命科学与工程学院 四川省天然药物仿生合成工程研究中心 成都 610031) \\ ( $b$ 伊犁师范大学 新疆天然产物化学与应用重点实验室 新疆伊宁 835000 )
}

\begin{abstract}
摘要 小檗碱(Berberine, BBR)是一种重要的天然药物, 是具有独特四环结构的异喹啉类生物碱. 近年来药理学研究发 现，小檗碱有望用于肿瘤及糖尿病等多发病的临床治疗. 结合Woodward 等提出的生源合成理论，综述了截至目前已报 道的小檗碱的全合成策略与方法.

关键词 天然药物; 小檗碱; 芐基异喹啉; 全合成
\end{abstract}

\section{Studies on the Chemical Synthesis of Natural Drugs Berberine}

\author{
Yan, Xifei ${ }^{a} \quad$ Zheng, Jianfeng ${ }^{*, a} \quad$ Li, Wei-Dong Z. ${ }^{*, a, b}$ \\ ( ${ }^{a}$ Sichuan Engineering Research Center for Biomimetic Synthesis of Natural Drugs, School of Life Science and Engi- \\ neering, Southwest Jiaotong University, Chengdu 610031)
}

$\left({ }^{b}\right.$ Key Laboratory of Natural Product Chemistry and Application in Xinjiang, Yili Normal University, Yining, Xinjiang 835000)

\begin{abstract}
Berberine is an important natural drug and a kind of benzyl isoquinoline alkaloid, which has unique four-ring structure. In recent years, pharmacological research has found berberine is expected to be used in the clinical treatment of cancer, diabetes and other frequently occurring diseases. Based on the Woodward's biosynthetic hypothesis, a schematic review on the synthetic studies towards berberine is provided.

Keywords natural drug; berberine; benzyl isoquinoline; total synthesis
\end{abstract}

早期，人们在小檗科灌木植物伏牛花(Damnacanthus indicus) 树皮中发现一种色素, 因其颜色泛黄, 广泛 用于羊毛、丝绸和皮革的染色. 1830 年, Buchner 等 ${ }^{[1-2]}$ 从伏牛花树皮中分离到了其结晶性化合物并命名为小 檗碱(Berberine, 1). 1984 年, Abadi 等 ${ }^{[3]}$ 通过 X 射线单晶 衍射等方法确证了硫酸小檗碱晶型的化学结构. 小檗 碱, 外观呈黄色针状晶体, 是一类具有双异喹啉骨架结 构生物碱, 多以稳定形式的盐酸盐存在.

小檗碱在中枢神经系统 ${ }^{[4]}$ 、心血管系统 ${ }^{[5-6]}$ 和运动 系统 ${ }^{[7]}$ 疾病中表现出良好的生物活性. 在我国, 小檗 碱存在于黄连及黄柏等多种传统中药中, 主要用于 治疗菌痢、胃肠炎和痛肿等细菌感染性疾病 ${ }^{[8]}$. 药理 研究表明, 其作用机制可能是选择性抑制细菌 RNA 转录、蛋白质/脂质的生物合成以及糖的酵解 ${ }^{[9]}$. 值得
注意的是，通过修饰芳环官能团取代基，丰富了小檗 碱骨架类型并扩大其作用范围 ${ }^{[10-13]}$, 如 Groenlandicine 2 对抑制 BACE1 酶 ${ }^{[14]}$ 有较高活性; Pseudoberberine 3 具有良好的降血脂作用 ${ }^{[15]}$; 化合物 4 具有良 好的抗肿瘤的生物利用度 ${ }^{[16]}$ (图 1).

目前自然界中发现的部分次生代谢产物如酚类、 萜类、甾体或生物碱只在特定种属植物中存在, 受光 照和温度等环境因素影响剧烈，需要多种酶或蛋白 共同参与才能长期稳定合成.

早期, 通过喂养实验，人们在金印草属北美黄连 (Hydrastis canadensis) ${ }^{[17]}$ 植物中发现了一系列合成小 檗碱的生物酶. 后来, 从多个种属 [如黄连属(Coptis

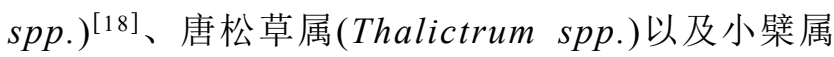
(Berberis spp.) $\left.)^{[19]}\right]$ 的不同植物(如黄连和三棵针)中均

\footnotetext{
*Corresponding authors. E-mail: jfzheng@swjtu.edu.cn; wdli@swjtu.edu.cn Received December 31, 2020; revised January 26, 2021; published online February 20, 2021.

Project supported by the National Natural Science Foundation of China (Nos. 21901215, 21672030), the Fundamental Research Funds for the Central Universities (No. 2682020CX53) and the Key Program of Natural Science Foundation of Xinjiang Autonomous Region Colleges (No. XJEDU2018I018) 国家自然科学基金(Nos. 21901215，21672030)、中央高校基本科研业务费专项资金(No. 2682020CX53)和新疆维吾尔自治区高校科研计划(重点)(No. XJEDU2018I018)资助项目.
} 


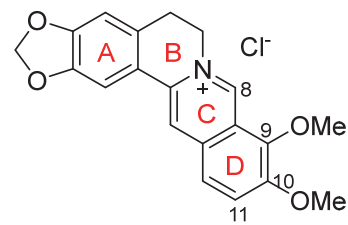

Berberine (1)

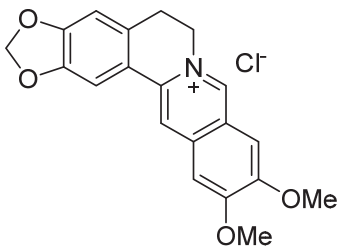

Pseudoberberine (3)

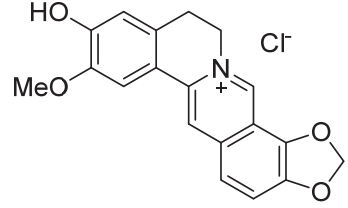

Groenlandicine (2)

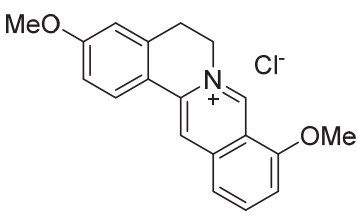

4
图 1 小檗碱及其类似物结构

Figure 1 Structures of berberine and its analogues

分离出小檗碱, 这说明了调控生物合成小檗碱的酶 或蛋白的基因簇具有很强分散性. 随着环境变化, 按 照生物进化规律, 该基因簇依然能长期稳定表达合 成小檗碱, 无论动植物或者其内生真菌如何进化, 其 控制合成小檗碱的能力并未消失或降低, 这些基因 簇可能已经具有一定普遍性, 广泛分布于不同植物 中.

被普遍认可的小檗碱生物合成主流途径是：以 $L$-酪氨酸为起始物, 分别转化为多巴胺和 4-着基苯乙 醛后, 经生物酶如合成酶及转移酶等作用合成骨架, 再经后期骨架修饰, 最终转化为天然小檗碱[2]. 值得 提出的是, 该途径是异喹啉生物碱的主要生物合成 途径(Scheme 1). 尽管该生物合成途径具有广泛性, 但它并不能清晰解释小檗碱也存在于其它种属植物, 如毛茛科莲花(Nelumbo SP.) $)^{[20]}$ 和罂粟科罂粟(Papaver somniferum L. $)^{[21]}$. 在这些植物中, 人们发现了小檗碱
类似物的生物合成途径，并没有发现小檗碱的生物 合成途径. 这些植物中可能存在小檗碱的其它生物 合成途径，其生物合成途径可能更加简洁高效，无需 几长的骨架搭建和结构修饰.

自然界中，单萜吲哚生物碱(monoterpene indole alkaloids) 是一类具有重要药用价值的天然产物. 其 生物合成途径被认为 ${ }^{[22]}$ 是从香叶醇出发, 经若干步 骤产生开环马钱子苷后，在异胡豆苷合酶催化下，其 与色胺缩合形成异胡豆苷. 随后，在不同酶的催化作 用下，单萜部分可能发生重排反应，形成形式各异的 单萜吲哚生物碱(Scheme 2, A). 尽管该路径可以清晰 地解释绝大部分单萜吲哚生物碱的生物合成, 但是, 并不能解释具有芳香性 $\mathrm{E}$ 环的单萜吲哚生物碱如 Alstoninline 的生物合成. 1948 年, Woodward 小 组 [23]提出单萜吲哚生物碱的生物合成假说, 包含 $\alpha / \beta$ 两 种类型 (Scheme 2, B). 其中 $\alpha$ 类型单萜吲哚生物碱的 生物合成途径被认为是植物体内存在的苯乙醛合成 子与色胺的 $\alpha$ 碳缩合后, 引入碳源, 经后期结构修饰 得到单萜吲哚生物碱化合物.

由于小檗碱与 $\alpha$ 类型单萜吲哚生物碱都具有芳 香性的 E 环，同时，小檗碱广泛存在于多种不同种属 的天然产物中. 因此, 我们推测, 在植物体内, 可能 存在两个已修饰的具有芳香性的合成子胡椒乙胺和 苯乙醛, 在酶的催化作用下, 缩合形成仲胺, 再引入 关键碳源，经温和氧化得到小檗碱(Scheme 2, C).

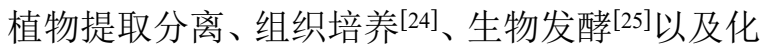
学合成 ${ }^{[26]}$ 是获取小檗碱的主要方式. 其中，化学合成 是最主要也是最直接的来源方式. 虽然早在 1967 年, Delgado 小组 ${ }^{[27]}$ 以别隐品碱(Allocryptopine)为原料, 通过半合成首次化学合成了小檗碱，然而直到 1969 年, Kametani 小组 ${ }^{[33]}$ 才首次完成了小檗碱的全合成.
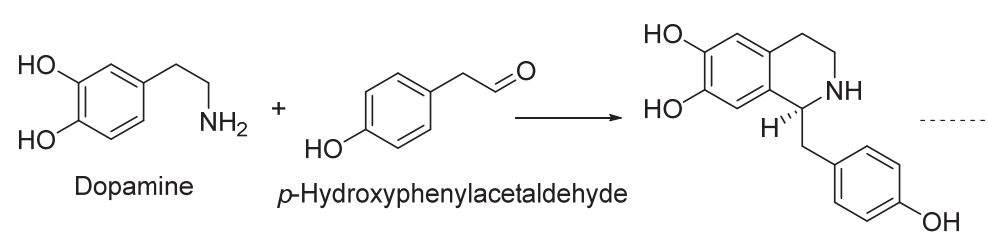

(S)-Norcoclaurine

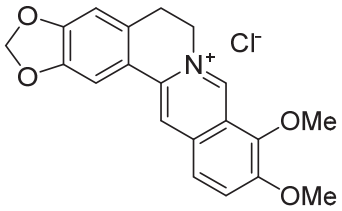

Berberine (1)

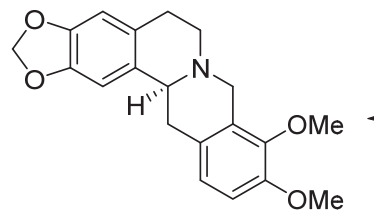

(S)-Canadin

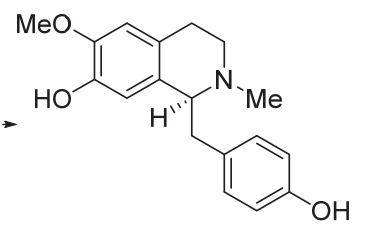

(S)-N-Methylcoclaurine

图式 1 小檗碱的生物合成途径

Scheme 1 Biosynthetic route of berberine 
(A)

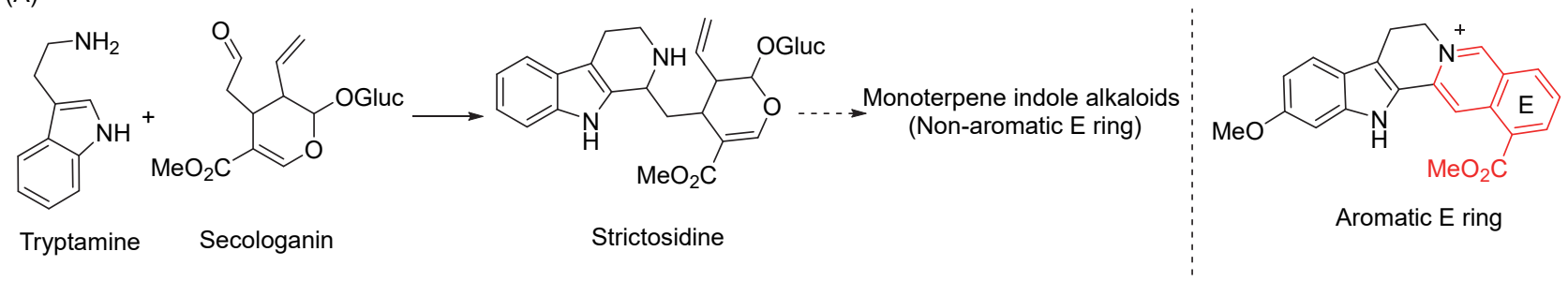

(B)

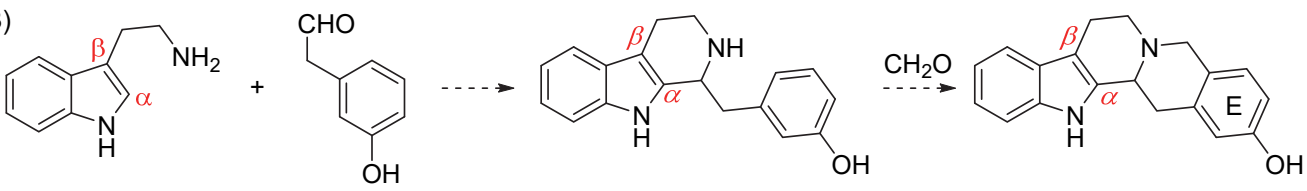

Tryptamine

Phenylacetaldehyde

(C)<smiles>NCCc1ccc2c(c1)OCO2</smiles>

Piperethylamine

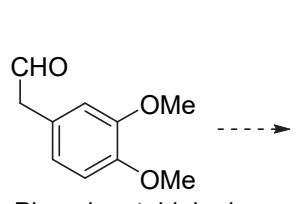

Phenylacetaldehyde

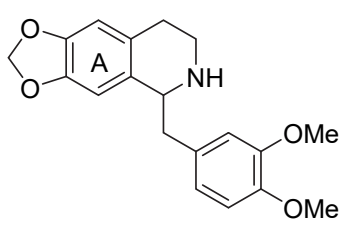

$[\mathrm{C}]$

[O]

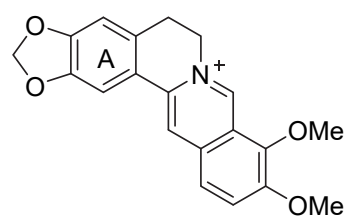

图式 2 主流的单萜吲哚生物碱生物合成途径、Robinson-Woodward 的单萜吲哚生物碱生物合成假说和小檗碱的生物合成猜想

Scheme 2 Major biosynthesis routes of monoterpene indole alkaloids, the Robinson-Woodward's biosynthetic hypothesis of monoterpene indole alkaloids and proposed major biosynthesis routes of berberine

同时，小檗碱的重要合成前体，四氢小檗碱 $(( \pm)-$ Canadine, 5)以及 8-氧代小檗碱(8-Oxoberberine, 6), 可分别通过氧化反应以及还原/氧化反应转化为天然 产物小檗碱.

鉴于此, 本文综述了从 1969 年以来发展的 8 条小 檗碱全合成路线以及 8 条前体化合物四氢小檗碱或 8-氧代小檗碱的合成路线. 策略上, 可分为仿生合成 策略(C1 环化法)、分子间偶联策略(C2 环化法)和其它 金属催化合成法三类. 最后, 总结了作者课题组在这 方面的工作. 下面, 将从这四个方面对文献进行回顾 (Scheme 3).

\section{2 仿生合成法(C1 环化法)}

仿生合成策略作为大自然在漫长进化过程中发 展出的高超合成策略和反应途径, 为合成化学家提 供了科学研究的一种重要思路. 从对自然界的思考 中提出问题、解决问题并进一步提出新的问题, 在这 一过程中化学家有机会利用实验室合成手段提出并 验证天然产物的生物合成途径, 有机会充分利用源 于底物本身的特性实现高效、富有艺术性的合成，同 时有机会发现自然界中尚未被发现的反应途径，扩 展新的化学领域.

最早于 1911 年, Pictet 小组 ${ }^{[28]}$ 以胡椒乙胺为最初 原料, 与取代苯乙酸发生酰胺化和 Bischler-
Napieralski 环化反应得到四氢异喹啉化合物 7, 该化 合物经 $\mathrm{NaBH}_{4}$ 还原并以甲醛为酰基化试剂和 $\mathrm{C}(8)$ 来 源, 合成氢化小檗碱. 然而, Buck 等[29-32]在对 Pictet 小组合成的小檗碱骨架分析时发现，由于区域选择 性控制，反应更利于得到 C(10), C(11)-二甲氧基氢化 小檗碱类似物 9, 并非 C(9),C(10)-二甲氧基氢化小檗 碱. 尽管 Pictet 小组并没有完成小檗碱的全合成, 但 其工作对后期小檗碱的生物途径研究以及全合成研 究产生了深远的影响(Scheme 4, A).

受小檗碱生物合成途径和 Pictet 小组研究工作的 启发, 1969 年, Kametani 等[33]首次报道了小檗碱的全 合成. 作者巧妙地通过在 D 环引入溴来控制甲醛甲 基化反应的区域选择性，成功实现了小檗碱的全合 成. 尽管合成步骤繁琐, 但这项工作为小檗碱全合成 奠定了重要基础. 作者以胡椒乙胺和溴代苯乙酸甲 酯为起始原料, 缩合得到酰胺化合物后, 经 BischlerNapieralski 环化反应得到化合物 10, 该化合物经还原 和水解得到溴代的四氢异喹啉化合物; 以甲醛为 $\mathrm{C}(8)$ 的来源，经脱溴反应、甲基化反应和氧化等得到 小檗碱天然产物 1 (Scheme 4, B).

2002 年和 2012 年, Schore 小组 ${ }^{[34]}$ 和 Mastranzo 小 组 ${ }^{[35]}$ 分别通过在芳基引入三甲基硅基和手性亚磺酰 基，以控制甲醛甲基化反应的区域选择性，最终完成 四氢小檗碱 $(S)$-Canadine (13)的合成(Scheme 4, C). 


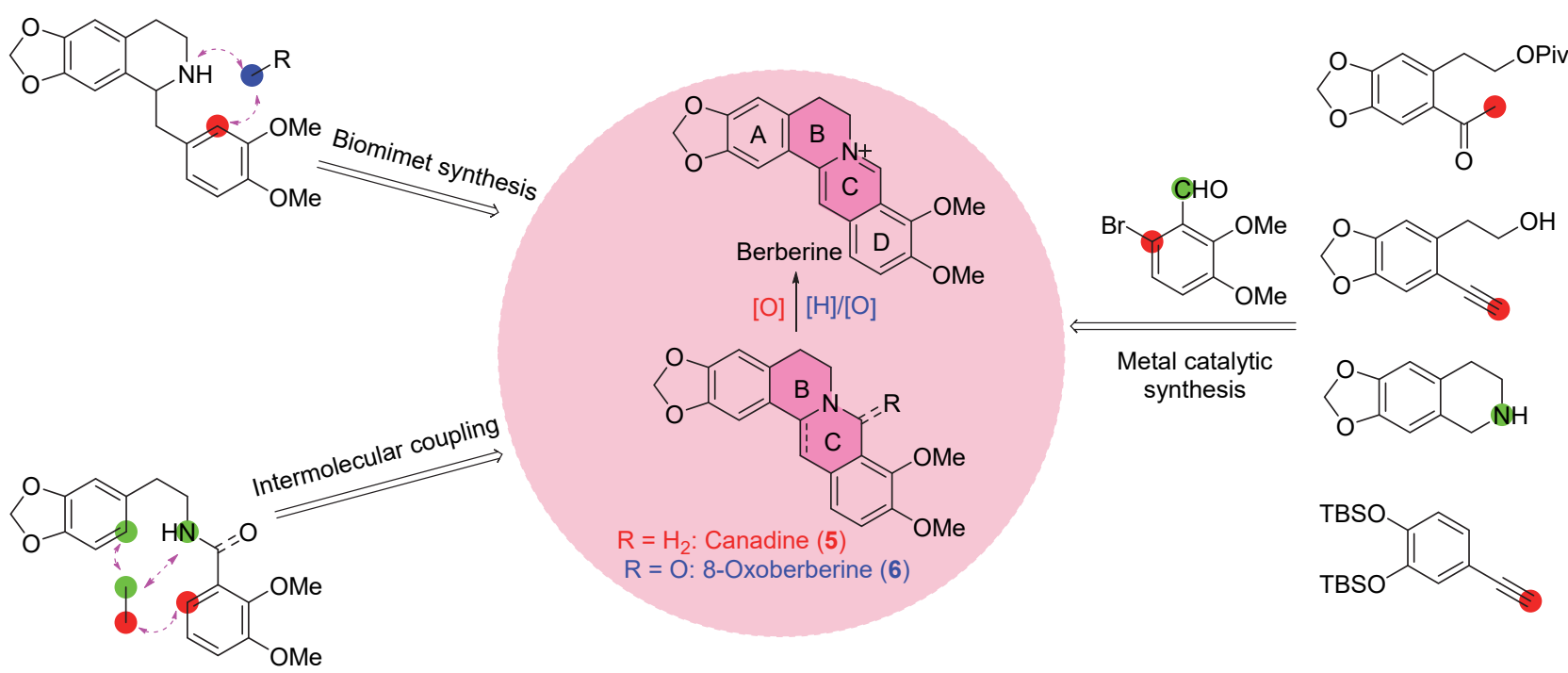

图式 3 小檗碱的全合成以及骨架合成策略

Scheme 3 Strategies of total synthesis or formal synthesis of berberine

(A) 1911 Pictet group
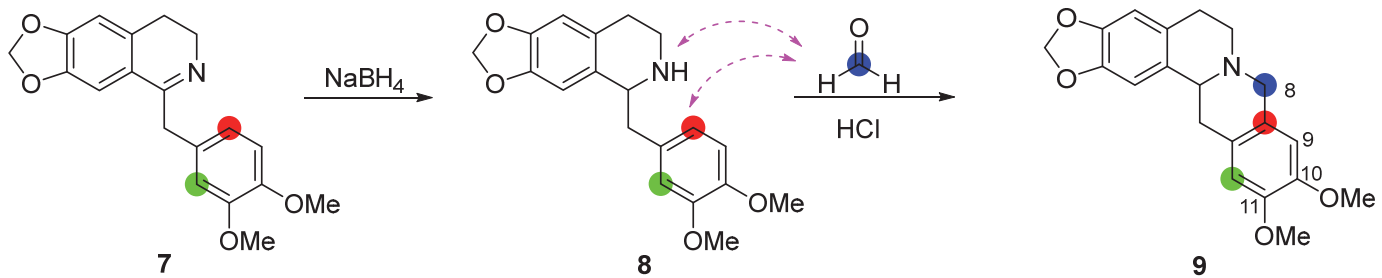

(B) 1969 Kametani group
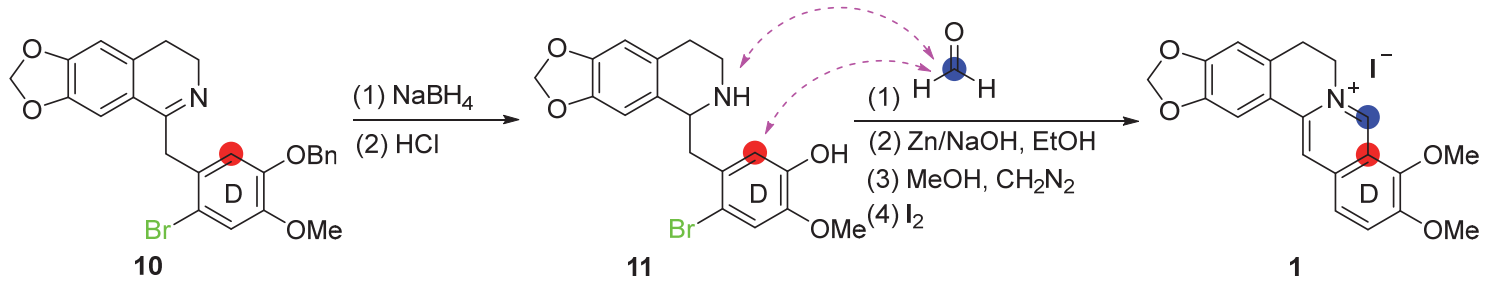

(C) 2002 Schore group; 2012 Mastranzo group

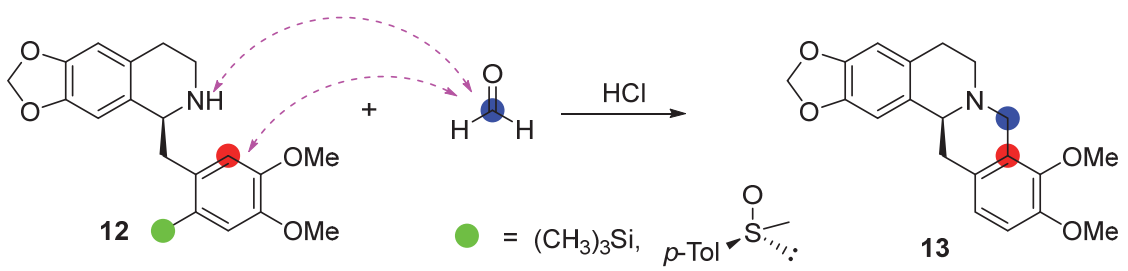

图式 4 甲醛参与的小檗碱骨架合成

Scheme 4 Synthesis of framework of berberine with formaldehyde

值得注意的是, 甲酰化试剂如乙酸酐 ${ }^{[36]} 、 N, N-$ 二 甲基甲酰胺 ${ }^{[37]}$ 以及一氧化碳 ${ }^{[38]}$ 等也常用于小檗碱骨 架的合成中(Scheme 5).

此外, 将含官能团的 $\mathrm{C}(8)$ 单元引入到 $\mathrm{D}$ 环, 利用 导向作用, 发生分子内环化反应构建 $\mathrm{C}$ 环, 完成小檗 碱及其骨架的合成，也具有重要的仿生学意义.

1996 年, Meyers 小组 ${ }^{[39]}$ 报道了四氢小檗碱的合 成. 首先, 在叔丁基锂的作用下, 手性甲脒化合物与
亲电试剂发生分子间 $\mathrm{S}_{\mathrm{N}} 2$ 反应，得到化合物 18. 随后， 在水合肼作用下，反应经脱甲脒和分子内酰胺化反 应得到 8-氧代小檗碱 19. 该化合物经 $\mathrm{LiAlH}_{4}$ 还原直 接得到四氢小檗碱. 与此同时, 手性甲榺化合物直接 经 $\mathrm{LiAlH}_{4}$ 还原可以形成氨基醇化合物 20, 在 $N$-溴代 琥珀酰亚胺(NBS)和二甲硫醚作用下，一步关环得到 四氢小檗碱(S)-(一)-Canadine (13) (Scheme 6, A, B). 

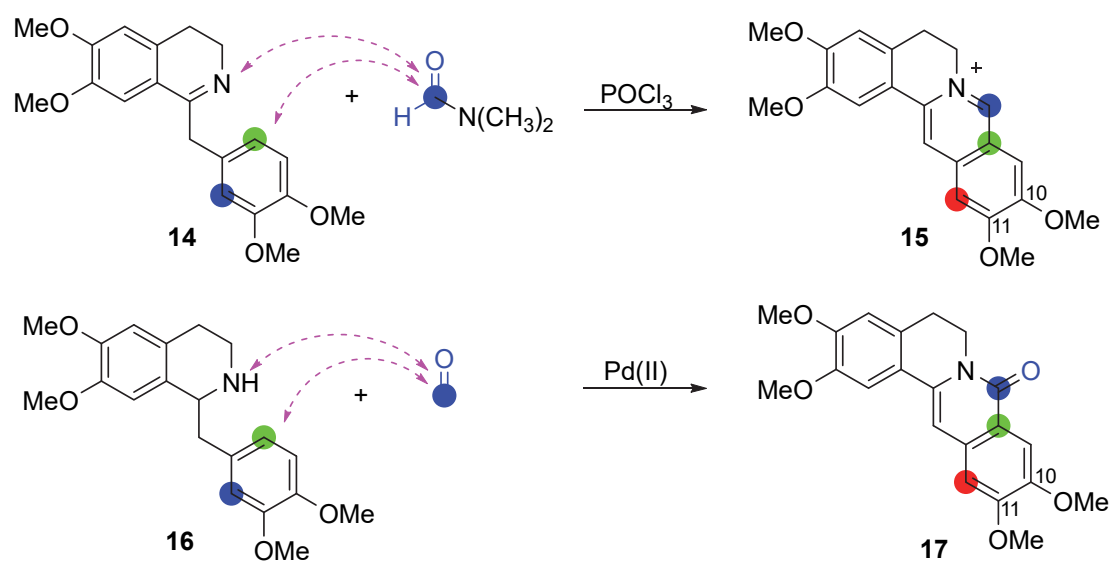

图式 5 其它甲酰化试剂参与的小檗碱骨架合成

Scheme 5 Synthesis of framework of berberine with other formylation reagent

(A) 1996 Meyers group<smiles>COc1ccc(CN2C(=O)Cc3cc4c(cc3[C@@H]2CC(=O)O)OCO4)cc1OC</smiles>

(B) 1996 Meyers group<smiles>COC(=O)c1cc(CC2c3cc4c(cc3CCN2C(=O)O)OCO4)ccc1OC</smiles>

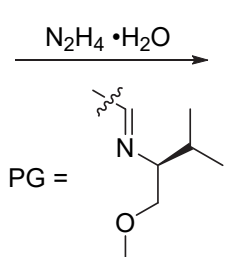

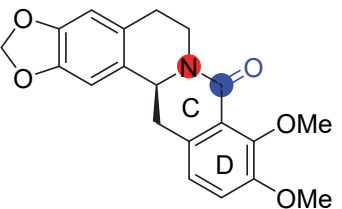

19

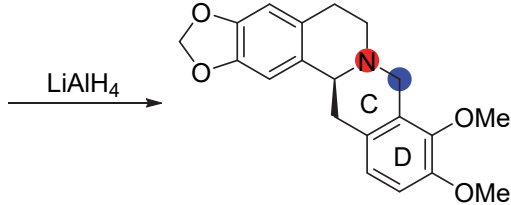

13

(C) 2007 Chen group

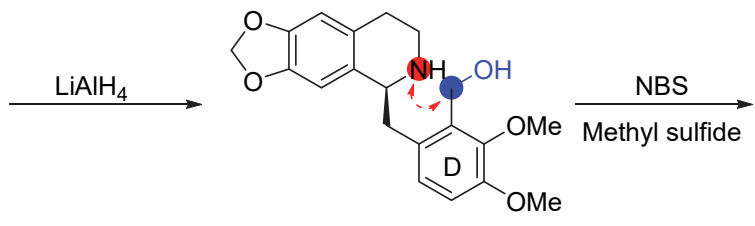

20<smiles>COc1ccc2c(c1OC)OC1Cc3cc4c(cc3C1CN2O)OCO4</smiles>

13<smiles>COc1ccc(CC(=O)NCCc2ccc3c(c2)OCO3)c(OC)c1OC</smiles>

21

(D) 2017 Lee group

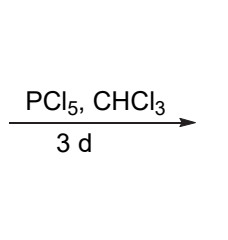

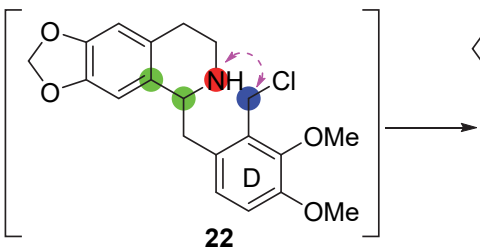

22

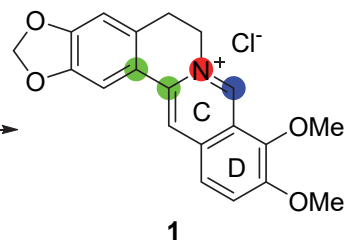

1

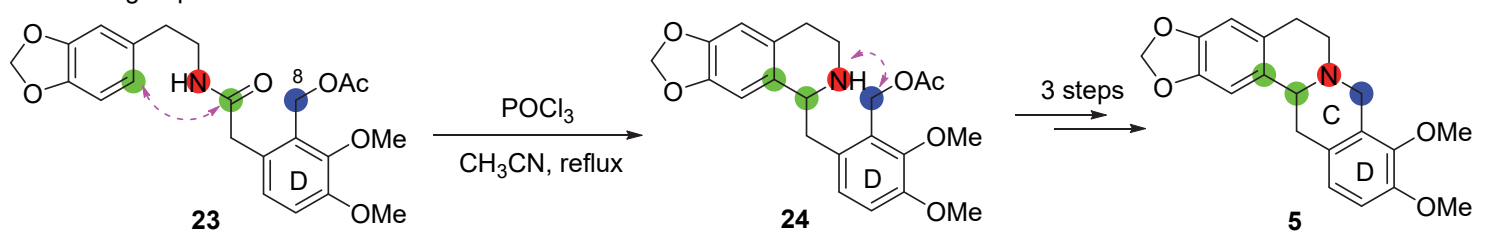

图式 $6 \mathrm{C} 1$ 分子内环化策略构建小檗碱及其骨架

Scheme 6 Synthesis of berberine and its framework using C1 intramolecular cyclization

2007 年, 陈再新小组 ${ }^{[40]}$ 报道了小檗碱的工艺合 成路线. 以商业可得的 2,3-二甲氧基苯甲醇和胡椒乙 胺为原料, 经多步合成包含小檗碱所有碳原子的酰 胺化合物 21. 在五氯化磷的作用下, 该化合物可能经
苄氯中间体，一步合成盐酸小檗碱化合物(Scheme 6 , C).

2017 年, Lee 小组 ${ }^{[41]}$ 报道了多种四氢小檗碱类似 物的合成. 以胡椒乙胺和 3,4-二甲氧基异色酮为原料, 
经两步反应合成酰胺化合物，该化合物经 BischlerNapieralski 环化反应合成四氢异喹啉化合物 24. 随 后，经多步反应，得到氢化小檗碱 5 (Scheme 6, D).

\section{3 分子间偶联策略(C2 环化法)}

以胡椒乙胺和邻香兰醛为原料, 经脱水缩合和 还原得到仲胺化合物，该化合物与 $\mathrm{C} 2$ 合成子发生分 子间环化反应，同时构建 $\mathrm{B}, \mathrm{C}$ 环，是小檗碱及其骨架 合成重要策略(Scheme 7).

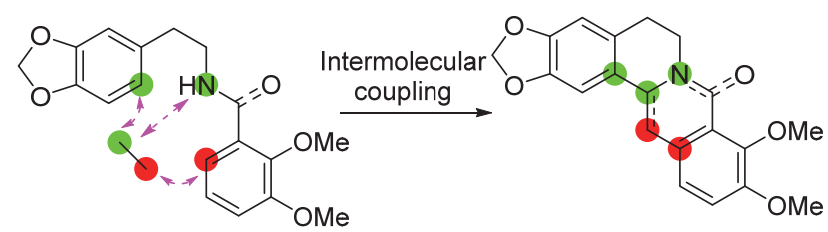

图式 7 C2 分子内环化策略小檗碱及其骨架

Scheme 7 Synthesis of berberine and its framework using C2 intramolecular cyclization

1972 年, 安静㜆小组 ${ }^{[42]}$ 以胡椒乙胺和邻香兰醛 为原料, 经脱水缩合和还原, 合成含有 $\mathrm{A} / \mathrm{D}$ 环的仲胺 化合物 25. 然后, 以乙二醛(26)为 $\mathrm{C} 2$ 合成子, 通过 傅-克反应等多步串联反应，同时构建 $\mathrm{B} / \mathrm{C}$ 环，完成 小檗碱的全合成. 值得提出的是, 该路线是首条符合
中国药典的工业合成路线. 由于反应过程存在高压 条件，金属镍催化氢化还原会给工业生产带来一定 的安全隐患. 基于此, 多个研究团队如冯豫川小组 [43]、广西南宁制药厂 [44]、东北制药厂 ${ }^{[45]}$ 、佑华制药(乐 山)公 司 ${ }^{[46]}$ 以及华中制药 ${ }^{[47]}$ 等通过使用 $\mathrm{KBH}_{4}$ 或水 合肼等还原体系改进了盐酸小檗碱的工业合成方法 (Scheme 8, A).

1987 年, Hanaoka 小组 ${ }^{[48]}$ 报道了四氢小檗碱的合 成. 以溴代胡椒乙胺和邻䔉芦醛为原料, 经脱水缩合 及 $\mathrm{LiAlH}_{4}$ 还原得到溴代仲胺化合物. 在温和条件下, 以 $\alpha$-甲硫基-乙酰氯 27 为 $\mathrm{C} 2$ 合成子，发生分子间偶 联反应，构建小檗碱 C 环，得到溴代酰胺化合物 29. 随后，在 Raney Nickel 作用下，化合物发生脱溴和脱 硫反应，最终经 Bischler-Napieralski 反应环化得到四 氢小檗碱 5 (Scheme 8, B).

1994 年, Nimgirawath 小组 ${ }^{[49]}$ 报道了小檗碱类似 物 8-氧代氢化小檗碱 $( \pm$ )-8-oxocanadine B 的合成. 作 者利用胡椒乙胺 $(30)$ 和乙酰氯(31)为原料合成了 1-甲 基-6,7-亚甲二氧基异喹啉，碱性条件下与溴代邻酰氯 32 酰化得到化合物 33, 再通过三正丁基氢化锡和偶 氮二异丁腈(AIBN)引发分子内的自由基环化完成了 (土)-8-oxocanadine (34) 的合成(Scheme 8, C).

(A) 1972 An group
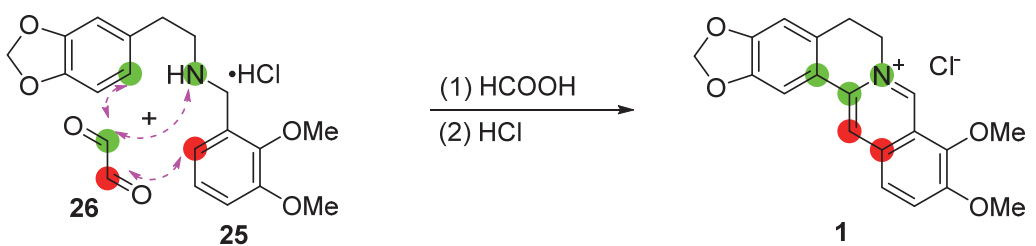

(B) 1987 Hanaoka group

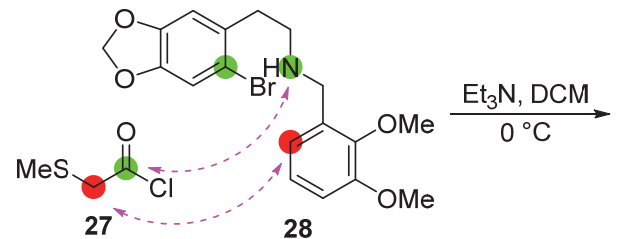

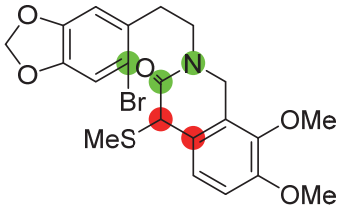

29

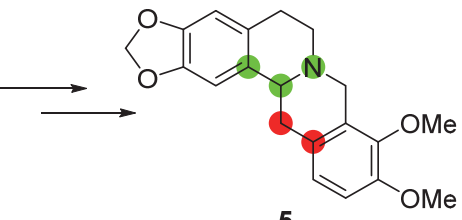

(C) 1994 Nimgirawath group

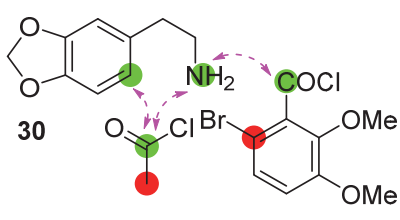

31

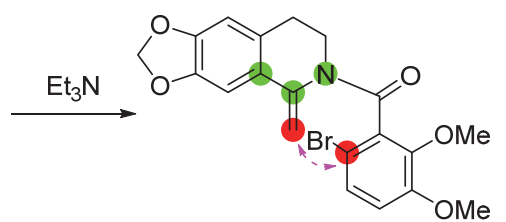

33

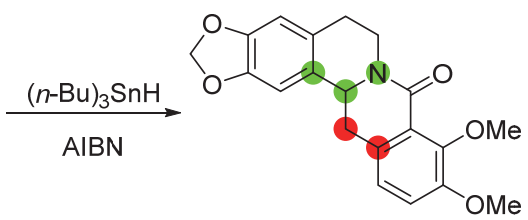

34

图式 8 安静䢞等小组全合成小檗碱及其骨架

Scheme 8 Total synthesis of berberine and its framework by An's group et al. 
2014 年, 吴勇小组 ${ }^{[50]}$ 报道了 8-氧代小檗碱的合 成. 以胡椒乙基溴(35)、2,2-二甲氧基乙胺(36) 以及 $2,3-$ 二甲氧基苯甲酸(37)为底物, 分别通过格式反应及 Pomeranz-Fritsch 反应等多步反应合成碘代化合物 38, 该化合物包含了小檗碱骨架所需的全部碳原子. 最 后经 $\mathrm{Pd}(\mathrm{OAc})_{2}$ 催化分子内 Heck 环化反应完成 8 -氧代 小檗碱 6 的合成(Scheme 9, A).

2016 年, Mhaske 小组 ${ }^{[51]}$ 报道了小檗碱类似物 8氧代氢化小檗碱的合成. 作者以胡椒乙胺和 2,3-二甲 氧基苯甲酸为原料, 经酰胺化以及碘代反应得到化 合物 39. 随后，以三丁基乙烯基锡(40)为 $\mathrm{C} 2$ 合成子, 在 $\mathrm{Pd}_{2}(\mathrm{dba})_{3}$ 的作用下, 发生 Stille 偶联反应得到化合 物 41. 在 $\mathrm{Pd}(\mathrm{OAc})_{2}$ 以及 $\mathrm{Cu}(\mathrm{OAc})_{2} \cdot \mathrm{H}_{2} \mathrm{O}$ 的条件下, 化 合物发生分子内的胺化反应以及 $\mathrm{C}-\mathrm{H}$ 键活化, 得到 ( \pm )-8-oxocanadine (34) (Scheme 9, B).

2018 年, Clift 小组 ${ }^{[52}$ 报道了小檗碱以及其类似物 的全合成. 以胡椒乙胺 32 和 2,2-二甲氧基乙酫(42)为 原料, 在三氟醋酸的作用下, 发生 Pictet-Spengler 反 应, 得到四氢异喹啉化合物; 该化合物与邻藜芦醛 (43) 缩合后经 $\mathrm{NaBH}(\mathrm{OAc})_{3}$ 还原得到叔胺化合物 44. 随后经 Friedel-Crafts 烷基化反应、消除反应以及氧化 反应, 一锅法得到盐酸小檗碱化合物. 该反应路线总 共为 4 步，总收率为 $54 \%$ (Scheme 9, C).

\section{4 其它金属催化合成法}

2014 年, Donohobe 小组 ${ }^{[53]}$ 以胡椒乙酸为原料, 经 羧酸还原、保护以及 Friedel-Crafts 酰基化反应得到化 合物 45. 同时, 溴代化合物 46 经乙二醇醛基保护得 到缩醛化合物后，在 $\mathrm{Pd}(\mathrm{II})$ 的作用下，化合物 $\mathbf{4 5}$ 与 $\mathbf{4 6}$ 发生氧化偶联反应得到化合物 47. 随后，化合物 47 发生水解反应构建小檗碱骨架 C 环. 值得提出的是, 特戊酯可以快速离去构建 $\mathrm{D}$ 环，作者在反应的水相 中观察到了小檗碱化合物. 随后，作者也尝试将 $\mathrm{Pd}$ 催化氧化偶联反应和水解反应一锅法，以 $28 \%$ 的总收 率分离得到了小檗碱化合物(Scheme 10, A).

2015 年, Anand 小组 ${ }^{[54]}$ 利用端炔化合物 49 和 46 经 Pd 催化作用发生 Sonagashira 偶联反应, 将小檗碱 的 $\mathrm{A}$ 环与 $\mathrm{D}$ 环相连得到化合物 $\mathbf{5 0}$. 随后发生 $\mathrm{Ag}$ 催化 的分子内环化反应得到异喹啉化合物; 最后, 在 $\mathrm{PPh}_{3}$ 的作用下, 发生取代反应得到小檗碱衍生物. 其工作 需要 4 步, 总收率为 $32 \%$ (Scheme 10, B).

2016 年, 童荣标小组 ${ }^{[5]}$ 以四氢异喹啉化合物 52、 端炔化合物 $\mathbf{5 3}$ 和溴代化合物 $\mathbf{4 6}$ 为起始原料, 在 $\mathrm{Cu}(\mathrm{I})$ 的作用下，一锅法将小檗碱的 $\mathrm{A} / \mathrm{B}$ 环与 $\mathrm{D}$ 环相连, 得 到化合物 54. 化合物 54 在 $\mathrm{Pd}(0) 、 \mathrm{Rh}$ 等多种过渡金 属催化下, 最后经还原、氧化反应得到小檗碱化合物 1. 其工作总共 6 步，总收率为 19\% (Scheme 10, C).

(A) 2014 Wu group
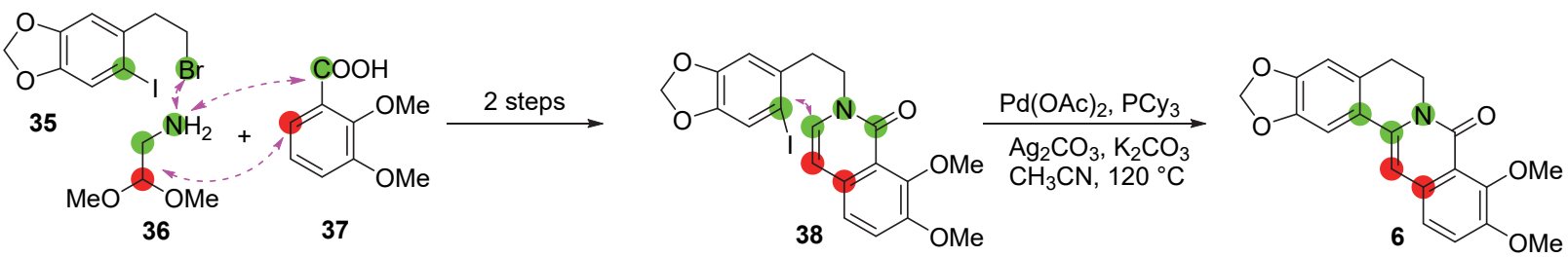

(B) 2016 Mhaske group

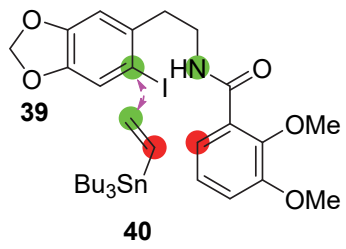

(C) 2018 Clift group

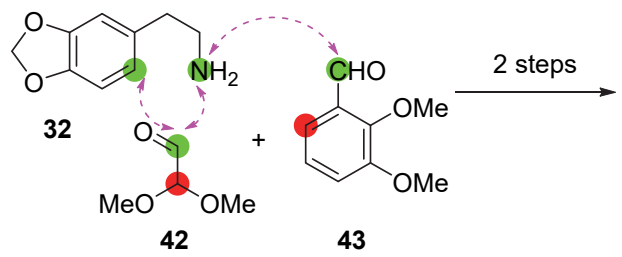

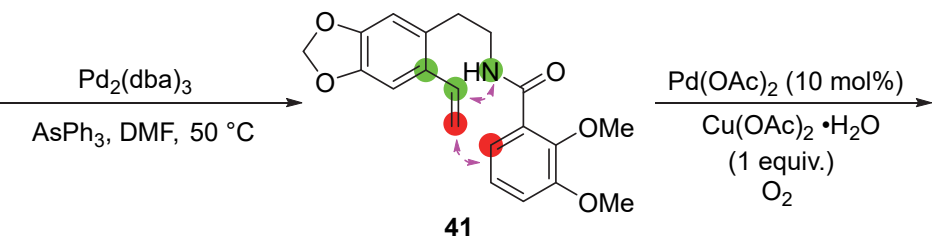

41

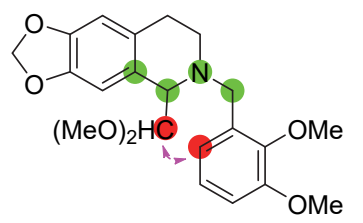

44
(1) $\mathrm{TfOH}, \mathrm{CH}_{2} \mathrm{Cl}_{2}$

(2) $\mathrm{I}_{2}, \mathrm{KOAc}, \mathrm{EtOH}$

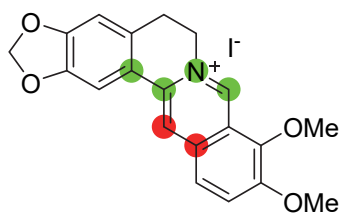

1

图式 9 小檗碱的骨架合成

Scheme 9 Synthesis of framework of berberine 
(A) 2014 Donohobe group<smiles>O=C(O)c1cc2c(cc1CCO[Pb])OCO2</smiles>

45

(B) 2015 Anand group<smiles>C#Cc1cc2c(cc1CCO)OCO2</smiles>

49

(C) 2016 Tong group<smiles>c1c2c(cc3c1OCO3)CNCC2</smiles>

52

(D) 2020 Zhang group

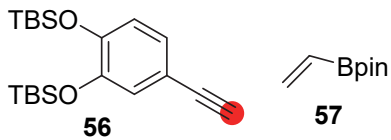

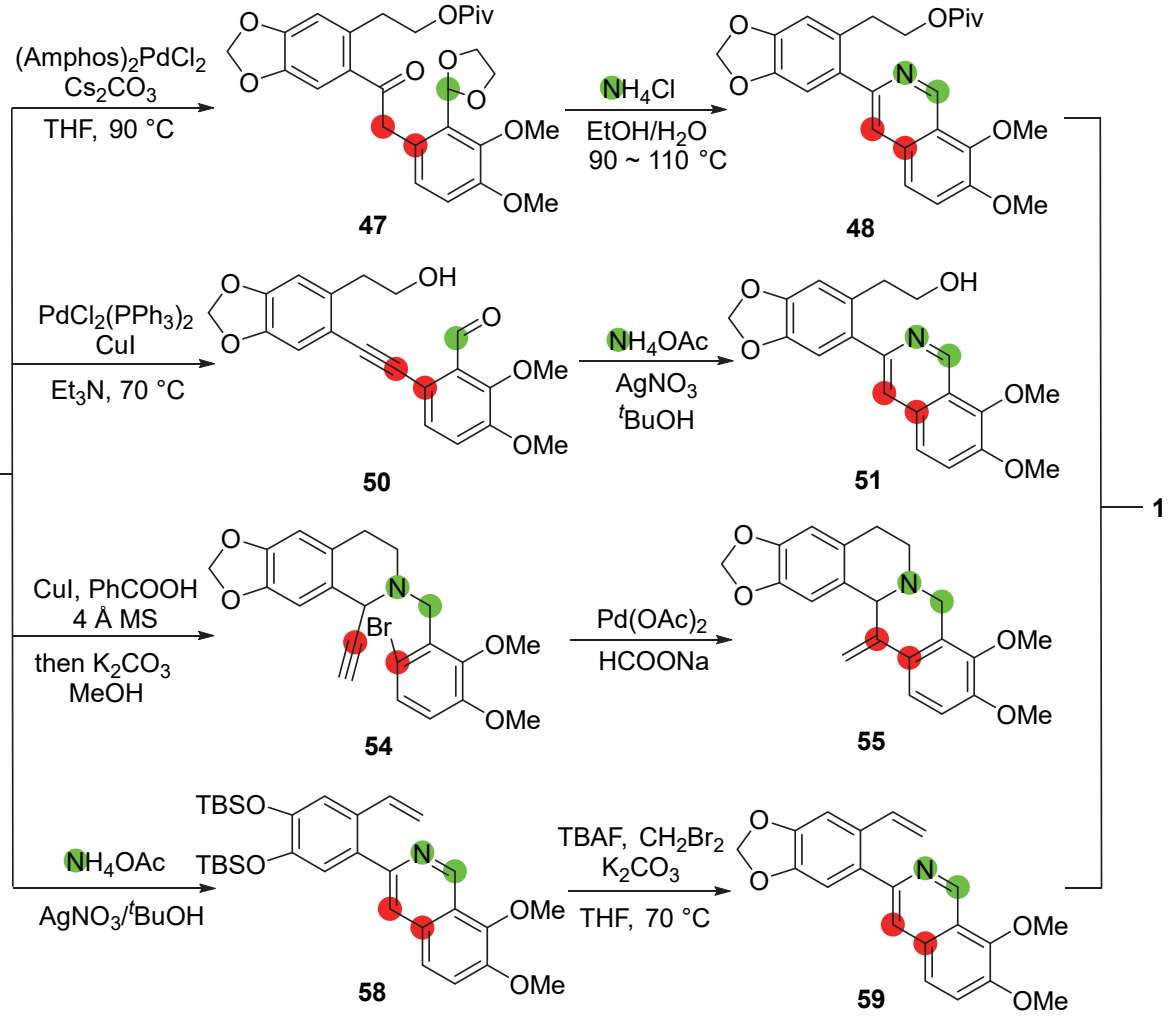

图式 10 金属催化全合成小檗碱

Scheme 10 Metal-catalyzed total synthesis of berberine

同年, 高栓虎小组 ${ }^{[56]}$ 利用分子内氢胺化及内酰化反 应合成了小檗碱类似物 Oxypalmatine.

2020 年, 张延东等 ${ }^{[57]}$ 以含保护基的苯乙炔 $\mathbf{5 6}$ 为 原料, 与溴代化合物 $\mathbf{4 6}$ 经 Sonogashira 偶联及 $\mathrm{Ag}$ 催 化的分子内环化得到异喹啉化合物; 随后经 $\mathrm{Rh}$ 催化 的 $\mathrm{C}-\mathrm{H}$ 乙烯基化并脱除叔丁基二甲基硅烷基后得到 化合物 59 , 再经过氮杂 $6 \pi$ 电环化反应, 实现了小檗 碱的 5 步全合成, 总收率为 7.6\% (Scheme 10, D).

\section{5 李卫东小组工作}

2005 年, 李卫东小组 ${ }^{[58}$ 在研究三尖杉碱全合成 过程中, 以四氢异哇啉 60 和溴代苯甲酸乙酯 $(61)$ 为原 料, 在强碱 ${ }^{\mathrm{B}} \mathrm{BOK}$ 作用下, 一步构建了小檗碱骨架化 合物 62, 再通过 $10 \% \mathrm{Pd}-\mathrm{C} / \mathrm{H}_{2}$ 还原反应和脱羒反应 ${ }^{[9]}$ 构建了 8-氧代小檗碱类似物 63 (Scheme 11). 应用该 策略, 我们成功实现了三尖杉碱及刺桐类生物碱[60] 的重要前体化合物的高效合成.

延续前期工作, 我们拟通过改进方法, 将其运用 到小檗碱及其类似物的全合成中, 目前, 已取得一定 的研究结果.

\section{6 总结与展望}

小檗碱, 作为经典的天然异喹啉类生物碱, 迄今为
止, 在临床上依然发挥着重要作用. 因其具有复杂的抗 菌作用机制以及多种生物活性, 已经得到了医药学界的 广泛关注. 明确其作用机制, 用于新的适应症也具重要 研究前景. 通过具有仿生合成策略的化学合成手段合成 天然产物, 对于阐述天然产物的生物合成有着重要的意 义, 不仅能进一步理解天然产物在植物体内的可能的合 成路线，同时通过仿生策略可能合成一些天然或非天然 的化合物, 为后续的药理研究以及药物篮选提供新的思 路. 毛茛碱是白毛莨植物中除小檗碱外分离得到的另一 类具有芳香性 $\mathrm{E}$ 环的异埕啉生物碱(图 2), 它们在生物 合成上可能有一定联系[1]. 研究表明, 其可以通过小檗 碱转化而来. 然而, 是否存在白毛莨碱 $[(-)-\beta$-hydrastine]转化为小檗碱的生物合成途径, 还有待未来进一步 研究与探索.

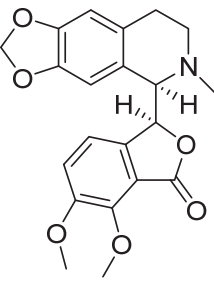

(一)- $\beta$-Hydrastine

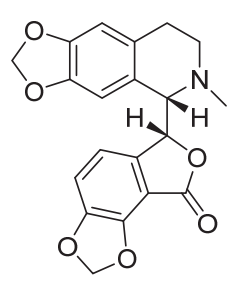

Bicuculline

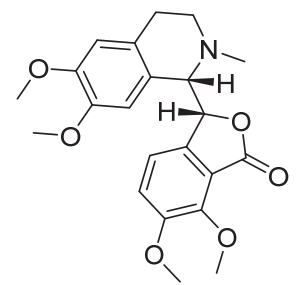

Cordrastine
图 2 白毛茛碱及其类似物结构

Figure 2 Structures of $(-)$ - $\beta$-hydrastine and its analogues 


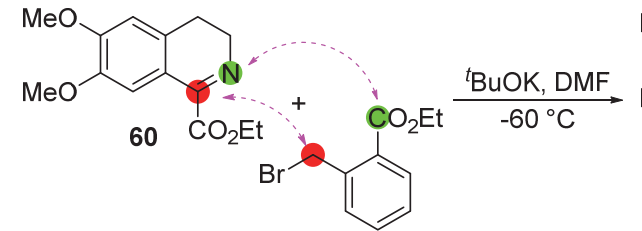

61

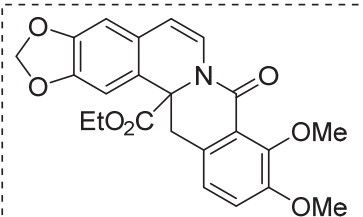

64

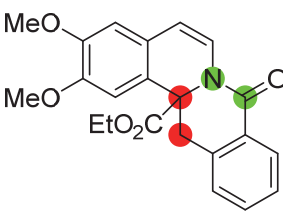

62<smiles>COc1ccc2c(C(=O)C(F)(F)F)c3n(c(=O)c2c1OC)CCc1cc2c(cc1-3)OCO2</smiles>

65
(1) $10 \% \mathrm{Pd}-\mathrm{C}, \mathrm{H}_{2}$

(2) Ref. [59]

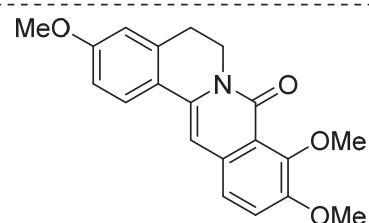

66

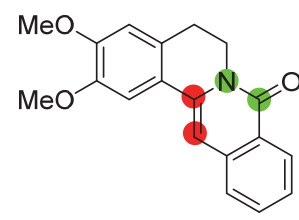

63

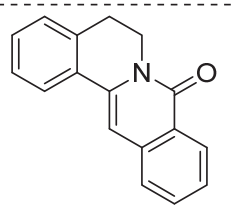

67

图式 11 李卫东小组的形式合成小檗碱骨架

Scheme 11 Formal synthesis of berberine skeleton by Li's group

截至 2020 年, 已经有许多课题组完成了小檗碱或 其前体化合物的合成(表 1). 自 1969 年 Kametani 小组首 次报道小檗碱的合成以来, 总共有 16 个小组发展了形 式多样的合成策略. 在这些策略中, 以芳香性 $\mathrm{A}$ 环和 $\mathrm{D}$ 环为合成子, 通过汇聚法构建骨架, 经后期修饰得到小
檗碱是研究最为广泛的. 其中, Clift 小组报道的以四步 反应 $54 \%$ 总收率合成小檗碱是最短的合成路线. 工业合 成中，安静妚小组报道的以胡椒乙胺和邻僽芦醛为原 料, 通过汇聚法合成小檗碱是目前最常用的方法之一. 利用 $\mathrm{Pd}$ 催化氧化偶联反应作为关键步骤合成小檗碱丰

表 1 小檗碱全合成研究工作总结

Table 1 Summary of total synthesis of berberine

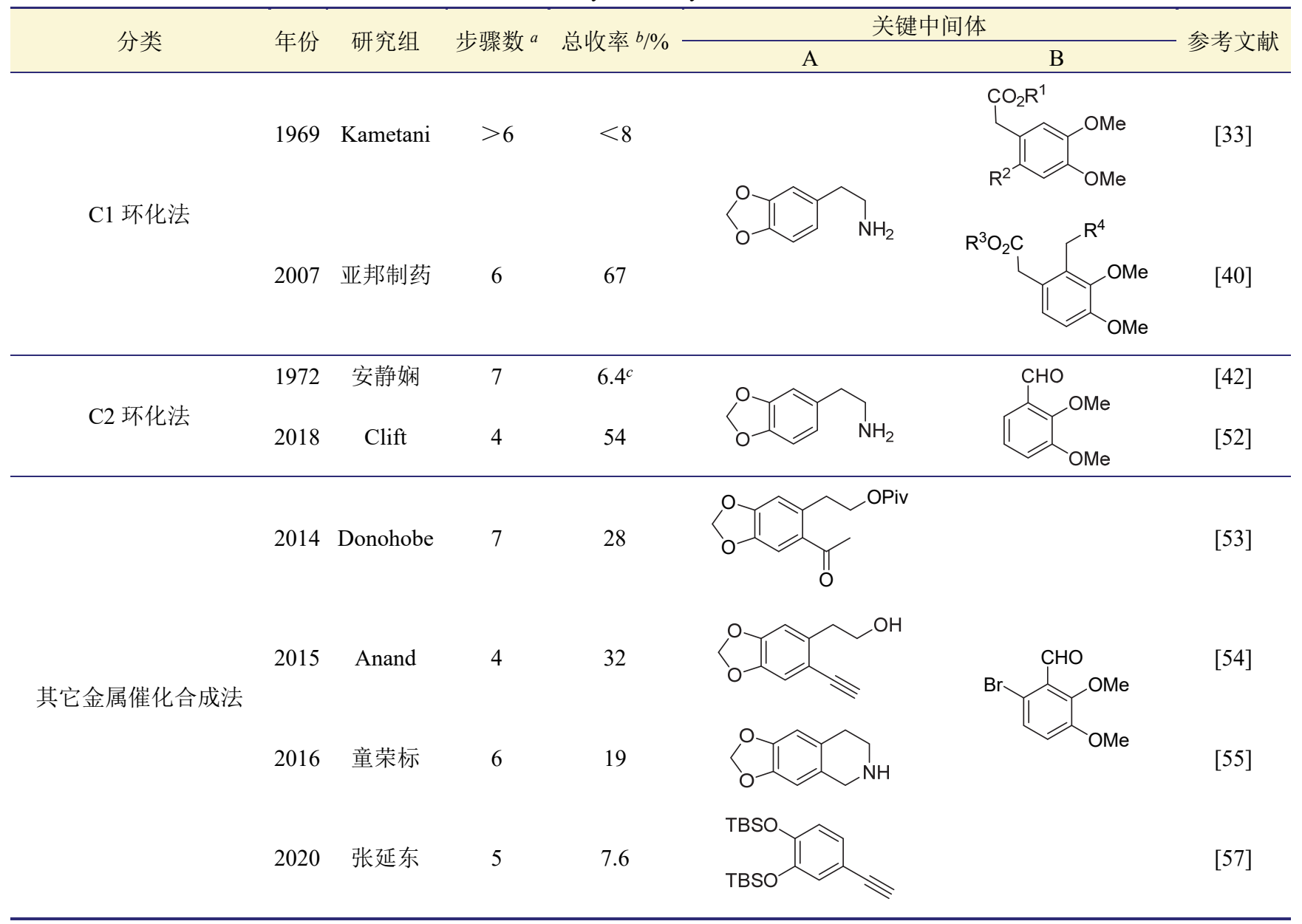

$\bar{a}$ 步骤数以文献报道计. ${ }^{b}$ 总收率以关键中间体计. ${ }^{c}$ 总收率以苯酚计. 
富了小檗碱的合成方法，但合成路线几长，贵金属催化 或使用剧毒品, 合成效率较低. 与此同时, 发展前体化 合物的合成策略为小檗碱的全合成提供给了新的思路. 李卫东小组通过改进瑞斯尔特合成法, 温和高效地构建 了小檗碱骨架，提高了合成效率，并有望将此方法运用 到小檗碱的全合成中.

目前, 我国小檗碱的年需求量达千吨级, 仅依靠提 取分离早已无法满足市场需求. 随着合成化学的快速发 展, 新的合成方法与策略层出不穷, 开发新型绿色高效 的合成策略具有重要意义.

\section{References}

[1] Schulz, E. R. J. Am. Pharm. Assoc. 1926, 15, 33.

[2] (a) Eckhardt, K.; Zeller, K.-P.; Siehl, H.-U.; Berger, S.; Sicker, D. Chem. Unserer Zeit 2017, 51, 344. (b) Buchner, J. A.; Herberger J. E. Repert. Pharm. 1830, 36, 1.

[3] Abadi, B. E. A.; Moss, D. S.; Palmer, R. A. J. Crystallogr. Spectrosc. Res. 1984, 14, 269.

[4] Durairajan, S. S. K.; Liu, L.-F.; Lu, J.-H.; Chen, L.-L.; Yuan, Q J.; Chung, S. K.; Huang, L.; Li, X.-S.; Huang, J.-D.; Li, M. Neurobiol. Aging 2012, 33, 2903.

[5] Hong, Y.; Hui, S.-C.; Chan, T.-Y.; Hou, J.-Y. Am. J. Chin. Med. 2002, 30, 589 .

[6] Kong, W. J.; Wei, J.; Abidi, P.; Lin, M.; Inaba, S.; Li, C.; Wang, Y. L.; Wang, Z. Z.; Si, S.Y.; Pan, H. N.; Wang, S. K.; Wu, J. D.; Wang, Y.; Li, Z. R.; Liu, J. W.; Jiang, J.-D. Nat. Med. 2004, 10, 1344.

[7] Lee, H. W.; Suh, J. H.; Kim, H.-N.; Kim, A. Y.; Park, S. Y.; Shin, C. S.; Choi, J.-Y.; Kim, J. B. J. Bone Miner. Res. 2008, 23, 1227.

[8] Xing, J.; He, H. World Latest Med. Inf. 2019, 19, 231 (in Chinese). (邢娟，何梦静，世界最新医学信息文摘, 2019, 19, 231.)

[9] Amin, A. H.; Subbaiah, T. V.; Abbasi, K. M. Can. J. Microbiol. 1969, 15,1067

[10] Cong, Z. Q. M.S Thesis, Shanghai Institute of Materia Media, Chinese Academy of Sciences, Shanghai, 2019 (in Chinese). (从湛青, 硕士论文, 中国科学院上海药物研究所, 上海, 2019).

[11] Liu, Y. Ph.D. Dissertation, East China Normal University, Shanghai, 2019 (in Chinese). (刘艺，博士论文，华东师范大学，上海，2019.)

[12] Sobolova, K.; Hrabinova, M.; Hepnarova, V.; Kucera, T.; Kobrlova, T.; Benkova, M.; Janockova, J.; Dolezal, R.; Prchal, L.; Benek, O.; Mezeiova, E.; Jun, D.; Soukup, O.; Korabecny, J. Eur. J. Med. Chem. 2020, 203, 112593.

[13] Zhang, L.; Shi, M. X.; Liu, J. F.; Han, W. N. J. Int. Pharm. Res. 2018, 45, 325 (in Chinese). (张龙, 史明星, 刘金风, 韩维娜, 国际药学研究杂志, 2018, 45, 325.)

[14] Jung, H. A.; Min, B.-S.; Yokozawa, T.; Lee, J.-H.; Kim, Y. S.; Choi, J. S. Biol. Pharm. Bull. 2009, 32, 1433.

[15] Li, Y.-H.; Yang, P.; Kong, W.-J.; Wang, Y.-X.; Hu, C.-Q.; Zuo, Z.-Y.; Wang, Y.-M.; Gao, H.; Gao, L.-M.; Feng, Y.-C.; Du, N.-N.; Liu, Y.; Song, D.-Q.; Jiang, J.-D. J. Med. Chem. 2009, 52, 492.

[16] Xu, B. B.; Jiang, X. J.; Xiong, J.; Lan, J.; Tian, Y.; Zhong, L.; Wang, X. Q.; Xu, N.; Cao, H. W.; Zhang, W. Q.; Zhang, H.; Hong, X. T.; Zhan, Y.-Y.; Zhang, Y. D.; Hu, T. H. J. Med. Chem. 2020, 63, 5841.

[17] Gear, J. R.; Spenser, I. D. Can. J. Chem. 1963, 41, 783.

[18] Muller, M. J.; Zenk, M. H. Planta Med. 1992, 58, 524.

[19] Hashimoto, T.; Yamada, Y. Annu. Rev. Plant Physiol. Plant Mol. Biol. 1994, 45, 257.

[20] Menéndez-Perdomo, I. M.; Facchini, P. J. Molecules 2018, 23, 2899.

[21] Beaudoin, G. A. W.; Facchini, P. J. Planta 2014, 240, 19.
[22] Wu, S. W.; Yang, M. Q.; Xiao, Y. L. Chin. J. Org. Chem. 2018, 38, 2243 (in Chinese).

(吴世文，杨盟权，肖友利，有机化学， 2018，38，2243.)

[23] Woodward, R. B. Nature 1948, 162, 155.

[24] Sato, F.; Yamada, Y. Phytochemistry 1984, 23, 281

[25] (a) Gao, Y.; Yin, H.; Sun Y. H.; Zhang, Z. Q.; Cui, Y. J. Fungal Res. 2008, 6, 216 (in Chinese).

(高杨，殷红，孙宇宏，张志强，崔迎，菌物研究，2008，6, 216.)

(b) Gao, Y.; Liu, M. Guangzhou Chem. Ind. 2012, 40, 91 (in Chinese).

(高杨, 刘铭, 广州化工, 2012, 40,91.)

[26] Liu, X. S. Hans J. Chem. Eng. Technol. 2020, 10, 306 (in Chinese). (刘雪松, 化学工程与技术, 2020, 10, 306.)

[27] Dominguez, X. A.; Delgado, J. G.; Reeves, W. P.; Gardner, P. D. Tetrahedron Lett. 1967, 8, 2493.

[28] Pictet, A.; Gams, A. Ber. Dtsch. Chem. Ges. 1911, 44, 2480.

[29] Buck, J. S.; Perkin, W. H. J. Chem. Soc., Trans. 1924, 125, 1675.

[30] Haworth, R. D.; Perkin, W. H.; Rankin, J. J. Chem. Soc., Trans. 1924, 125, 1686.

[31] Buck, J. S.; Perkin, W. H.; Stevens, T. S. J. Chem. Soc., Trans. 1925, 127, 1462.

[32] Buck, J. S.; Davis, R. M. J. Am. Chem. Soc. 1930, 52, 660.

[33] Kametani, T.; Noguchi, I.; Saito, K.; Kaneda, S. J. Chem. Soc. C 1969, 2036.

[34] Cutter, P. S.; Miller, R. B.; Schore, N. E. Tetrahedron 2002, 58, 1471.

[35] Mastranzo, V. M.; Romero, J. L. O.; Yuste, F.; Ortiz, B.; Sánchez-Obregón, R.; García Ruano, J. L. Tetrahedron 2012, 68, 1266.

[36] Makhey, D.; Gatto, B.; Yu, C.; Liu, A.; Liu, L. F.; LaVoie, E. J. Biorg. Med. Chem. 1996, 4, 781 .

[37] Szawkalo, J.; Czarnocki, Z. Monatsh. Chem. 2005, 136, 1619.

[38] Miyazawa, M.; Tokuhashi, T.; Horibata, A.; Nakamura, T.; Onozaki, Y.; Kurono, N.; Senboku, H.; Tokuda, M.; Ohkuma, T.; Orito, K. J. Heterocycl. Chem. 2013, 50, E48.

[39] Matulenko, M. A.; Meyers, A. I. J. Org. Chem. 1996, 61, 573.

[40] Chen Z. X.; Tao, F.; Xia, Z. J.; Ma, S. M. CN 101245064, 2008

[41] Lee, D. Y. W.; Liu, J.; Zhang, S. Z.; Huang, P.; Liu-Chen, L.-Y. Bioorg. Med. Chem. Lett. 2017, 27, 1437.

[42] North-east Pharmaceutical Factory Chin. J. Pharm. 1972, (4), 4 (in Chinese).

(东北制药总厂，中国医药工业杂志, 1972, (4), 4.)

[43] (a) Chen, C.; Luo, Z. M.; Yang, H. J.; Feng, Y. C. Chin. J. Org. Chem. 2016, 36, 1426 (in Chinese).

(陈程, 罗卓玛, 杨鸿均, 冯豫川, 有机化学, 2016, 36, 1426.)

(b) Chen, C.; X, M. M.; Zhao, Q.; Liu, C. X.; Yang, H. J.; Feng, Y. C. Chin. J. Org. Chem. 2017, 37, 503 (in Chinese).

(陈程, 徐蒙蒙, 赵青, 刘承秀, 杨鸿均, 冯豫川, 有机化学, 2017, 37, 503.

[44] Guangxi Nanning Pharmaceutical Factory Chin. J. Pharm. 1973, (7), 4 (in Chinese).

(广西南宁制药厂，中国医药工业杂志，1973，(7)，4.)

[45] Chen, S. Q.; Lin W. F.; Zang, J. H.; Liu, J. H.; Qi, S. H.; Zhang, L. Q.; Song, G. L. CN 1312250, 2001.

[46] Tang C. G. CN 106543171, 2017.

[47] Feng, X.; Fu, L.; Zhao, C. A. CN 107868072, 2018.

[48] Yasuda, S.; Hirasawa, T.; Hanaoka, M. Tetrahedron Lett. 1987, 28, 2399.

[49] Nimgirawath, S.; Ponghusabun, O. Aust. J. Chem. 1994, 47, 951.

[50] He, Y.; Zheng, Y.; Hai, L.; Wu, Y. Chin. J. Chem. 2014, 32, 1121.

[51] Garad, D. N.; Mhaske, S. B. Org. Lett. 2016, 18, 3862.

[52] Mori-Quiroz, L. M.; Hedrick, S. L.; De Los Santos, A. R.; Clift, M. D. Org. Lett. 2018, 20, 4281.

[53] Gatland, A. E.; Pilgrim, B. S.; Procopiou, P. A.; Donohoe, T. J. Angew. Chem., Int. Ed. 2014, 53, 14555.

[54] Reddy, V.; Jadhav, A. S.; Anand, R. V. Org. Biomol. Chem. 2015, $13,3732$.

[55] (a) Zhou, S. Q.; Tong, R. B. Chem.-Eur. J. 2016, 22, 7084 
(b) Yu, J. X.; Zhang, Z. H.; Zhou, S. Q.; Zhang, W.; Tong, R. B. Org. Chem. Front. 2018, 5, 242.

[56] Li, K.; Ou, J. J.; Gao, S. H. Angew. Chem., Int. Ed. 2016, 55, 14778.

[57] Jiang, X. J.; Zeng, Z. X.; Hua, Y. H.; Xu, B. B.; Shen, Y.; Xiong, J.; Qiu, H. J.; Wu, Y. F.; Hu, T. H.; Zhang, Y. D. J. Am. Chem. Soc. 2020, 142,15585
[58] Li, W.-D. Z.; Yang, H. Tetrahedron 2005, 61, 5037.

[59] Reimann, E.; Grasberger, F.; Polborn, K. Monatsh. Chem. 2003, 134, 991.

[60] Guo, W.-S. Ph.D. Dissertation, Nankai University, Tianjing, 2012 (in Chinese) (郭维斯，博士论文，南开大学，天津，2012.)

[61] Spenser, L. D.; Gear, J. R. J. Am. Chem. Soc. 1962, 84, 1059.

(Zhao, C.) 